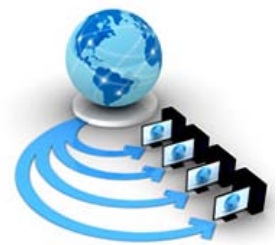

Volume 9, No. 2, March-April 2018

International Journal of Advanced Research in Computer Science

RESEARCH PAPER

Available Online at www.ijarcs.info

\title{
IAODV: AN IMPROVED AODV ROUTING PROTOCOL FOR MANET
}

\author{
Madhup Shrivastava \\ Department of Computer Technology and Application \\ NITTTR Bhopal (M.P.) India \\ M.A. Rizvi \\ Department of Computer Technology and Application \\ NITTTR Bhopal (M.P.) India
}

\author{
Monika Sahu \\ Department of Computer Science and Engineering \\ Govt. Polytechnic College Dindori (M.P.), India \\ Khaleel Ahmad \\ School of Computer Science and Information Technology \\ Maulana Azad National Urdu University Hyderabad, India
}

\begin{abstract}
Mobile Ad hoc Network is a network of independent nodes, which are mobile, connected through the wireless medium and highly dynamic by nature, and exemplified by the absence of physical infrastructure. The diversity and variations in wireless mobile devices are generating the revolutionary changes in the way users are using the services and resources for their purposes, at the same time it also poses many challenges to the researchers. In this paper, authors contribute toward the design of a new adaptive Quality of Service (QoS) paradigm for MANET. An honest attempt is made to develop an approach to decrease the overall delay, i.e. End-to-End delay and increase the efficiency of Protocol and also tried to make it more reliable by increasing the Packet Delivery Ratio (PDR) by applying Dijkstra Algorithm (for finding shortest and optimal route) and reducing queuing delay for increasing throughput by using a new hybrid algorithm. The modified AODV, which is named as Improved AODV (IAODV), enhance the "Packet Delivery Ratio" and reduce the "Queuing Delay".
\end{abstract}

Keywords: MANET, AODV, Improved AODV (IAODV), QoS, Packet Delivery Ratio (PDR), End to end Delay

\section{INTRODUCTION}

In modern times wireless networks have made great evolution towards mobile and wireless communication. One noteworthy point to be noted of such networks is that they are infrastructure less and self-configuring network [1]. MANET is basically a connectionless network with no centralized control, no fixed or a static infrastructure and high mobility [2]. It is a network with mobile routers which are linked through wireless links, the combination of which can form a capricious arrangement of nodes or a topology. The routers are in open and can move capriciously and organize themselves capriciously; as a result, wireless network topology could change dynamically and spontaneously. MANETs are typically set up in conditions of crisis for provisional services or merely if there are no resources available to set up the complex networks. MANET can function without any fixed infrastructure, which makes them trouble-free to set up. Because of the insufficiency of any constant resources, i.e. fixed infrastructure, it is difficult to use already active routing protocol for MANET services, and it creates a number of confronting in ensuring the protection of the messages due to the clashes between the requirements of network security and the demands of mobile networks[3], refer to Fig 1.

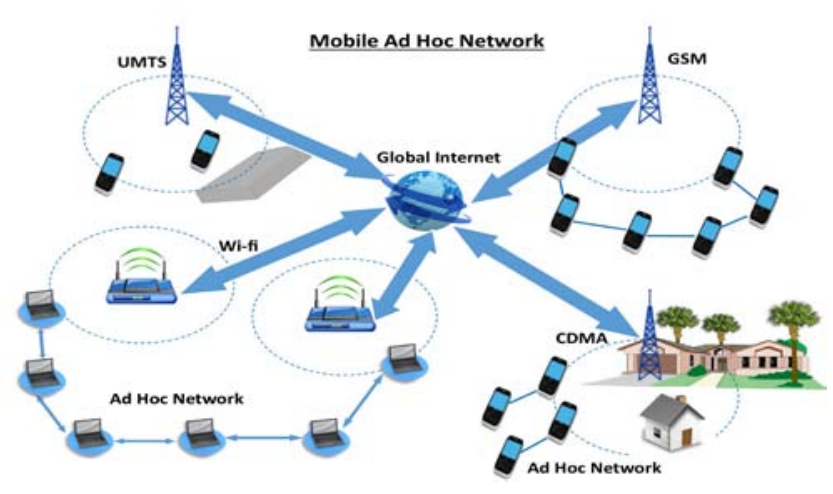

Fig 1: MANET

Infrastructureless communication in MANET causes many challenges and issues in the researcher's community in the context of improving the quality of services (QoS), the level of reliability etc. within the restrictions of narrow resources. The overall performance determines, is being tested on various protocols on different network circumstances, verified the honesty of the routing schemes in addition to their restrictions [4]. In recent technology epoch, the attempt of MANET is to endow with the capability of wireless communication by adapting ad hoc routing protocol's functionality in nodes. The mobile nodes are frequently changing its position in the network resulting in dynamic topology, which makes routing a complicated task [5]. MANET does not require any fixed or permanent infrastructure to be deployed, for example, base stations, etc. Hence, it is an alternative networking technology for connecting mobile devices expediently and impulsively [6].

Wireless communication has some limitations like bandwidth which needs proper management to achieve the requirement. The demand for high bandwidth and large storage is also a very big challenge of this technological world. To face these challenges a multi-tier framework is introduced 
which consist of various technologies i.e. IoT [7]. The Internet of Things is the combination of various networking devices like vehicles, buildings and other devices those are electronic, software or other network connecting device which permits gathering and interchanging of data [8]. These devices communicate using the internet and updating their data from the cloud or a server. In future time the technology gets popular and it will be the part of our day to day lives [9].

\section{TYPES AND FEATURES OF MANET}

- VANETs are familiarized devices which are fitted with the cars and roadside devices. The buses, vans or any other vehicle moving and changing locations in the city limits to lead up students, and accomplish this network. This can solve the problem of tracking the vehicle easily.

- VANETs are friendly of bogus intellect that helps the vehicle to become intelligent and avoid vehicle-to-vehicle collisions even in busy traffic conditions.

- Based on Internet hotlink MANET or Adaptable ad-hoc Networks (iMANET) with various mobile nodes and anchored Internet Gateway nodes. In this such splash of networks familiarized mobile ad hoc acquisition protocol does not administer directly [10].

The major features of the MANET are:

- Dynamic Topologies- Mobile nodes' movement is unpredictable; in this case, the network topology changes selfassertion and rapidly indiscriminately times.

- Bandwidth Limitations - Wireless connectivity has substantially brought down bandwidth limit as compared with their hardwired counterparts. Furthermore, the perceived quality of service parameter for e.g. throughput is very less in wireless based communication than that of the radio's maximum transmission rate, in view of lessened, clamor, interference circumstances and so forth.

- Energy-constrained - Most of the mobile network nodes are using chargeable batteries or other chargeable devices for their source of energy. The most important criteria for these nodes, is that the system should be designed in a way that should use less energy.

- Physical Security Limitations - Nodes of the mobile ad-hoc network are more probable inclined to physical security menace as compared to wired networks. The expanded probability of listening stealthily, spoofing, and some other ambush like denial-of-service attacks should be taken care off. - Scalability - A few predicted networks might be bulky, for example, 10 or 100 nodes only in the specified routing area. The requirement for adaptability is not exclusive to MANET, but sometimes it is the need of the circumstances and their characteristics, it is a whole lot tougher to attain scalability.

\section{ADHOC ON DEMAND DISTANCE VECTOR ROUTING}

It is the very commonly used the protocol in various fields of wireless communication. In this protocol the broadcast message is sent to all the neighboring nodes for sending a message to the pre-decided destination. An acknowledgment is sent to all the nodes which are transmitting messages from the node which is sourced node, now source node makes a decision to transmit the data packets depending upon the returned values from different nodes. If any link failure is found, this message will be broadcasted and replicates the same for successful transmission [11]. The working of AODV is partitioned into two stages:
- Discovery of Route

- Maintenance of Route

\section{ISSUES AND CHALLENGES IN MANET}

Nodes work with shared wireless transmission medium; the topology of the network transforms randomly and very animatedly; radio link connectivity dependability is an issue; frequent breakdown occurs; there is no immobile infrastructure etc. An environment of MANET has to overcome certain issues of inadequacy and constraint. These are as follows:

- The characteristic of wireless link varies with respect to time

- Wireless transmission range is limited

- Packet losses due to errors in transmission

- Route changes because of mobility

- Frequent network partitioning

- It confronts a lot of security menaces just like wired networks, which includes DoS, DDoS, RDDoS, spoofing, eavesdropping and other threats too. These attacks are mostly categorized on the premise of utilizing techniques and the outcomes [12].

\section{A. Quality of Services (QoS)}

In networking QoS is used which defined and maintained the quality of services over the network. QoS is the capacity to define a priority to various applications, users, or data flows, or guarantee a specific echelon of the performance of data movement in the network. A lot of activities can be done with the packets as they move through the network from source to destination. Some of the following problems are observed:

- $\quad$ End-to-End Delay (total time taken by a packet to travel from source to destination)

It can be calculated mathematically below:

E2E Delay $=\sum$ (arrival time - send time) $\sum$ Number of connections

- $\quad$ Packet Delivery Ratio (PDR)

Sometimes router fails to deliver some of the packets because of connection loss or they reach when the buffers are as of now full.

PDR = Sum of Number of packets received / Sum of Number of packets sent

- Throughput

Throughput is the ratio of output with respect to input because of the differing load of the users sharing the similar network resources, known as bit rate (it can be defined as maximum throughput).

- Errors

Occasionally packets are destroyed due to bit errors caused by clamor and obstruction, in wireless communication, particularly; these are called the error in messages when the transmission is done over the network.

- $\quad$ Latency

Latency means the delayed or less long time for each packet which it takes to arrive the destination.

- $\quad$ Out-of-order delivery

When an assortment of interrelated packets is routed through diverse routes in a network of diverse packets to reach the destinations, each resulting in a different delay because of this packet arrives at the destination in a different order in the manner in which they were sent [13].

After studying all the concepts of MANET many deficiencies are found in it and because of these problems the 
efficiency of the total network is degrading continuously. The problems are like:

- Problem of End-to-End Delay

- Problem of dropped packet

- The problem of throughput etc.

These all problems affect the competency in the network.

In this paper an attempt is made to deal with these problems in the network, which is used AODV protocol of Mobile Ad hoc networking. An attempt is made to establish a network with less End-to-End Delay and high packet delivery ratio, which jointly increase the overall throughput of the network, so the efficiency will automatically increase.

\section{END TO END DELAY AND PACKET DELIVERY RATIO}

\section{A. End to End Delay}

This may also be outlined as the total time taken for a packet to travel or transmitted over the network to reach the destination.

Mathematically End-to-End Delay is shown below:

$d e 2 e=N[d t r+d p+d p r o]$

Where,

de2e = end-to-end delay

$\mathrm{dtr}=$ delay in Transmission

$\mathrm{dp}=$ delay in propagation

dpro = delay in processing

$\mathrm{N}=$ number of links to the destination(Number of routers +1 )

\section{a) Transmission delay}

Delay occurred to Store-and-forward or transmission delay can be defined as the total time taken to send the data over the communication channel when packet switched network is used. This is the delay which is caused by the data-rate of the link. This delay can be explained, it is proportional to the packet's length in bits.

This is explained by the given formula:

$d t r=N b / R$

Where,

dtr is the delay in transmission

$\mathrm{Nb}$ can be explained a number of bits, and

$\mathrm{R}$ can be explained as transmission rate (says in bits/second)

\section{b) Propagation delay}

In computer networks, propagation delay can be explained as the total time taken by data to travel from the source to the destination. It can be calculated as the ratio between the length of the route to that of the link and the speed of propagation over the specific communication medium.

Propagation delay is $d p=D i / S p$

where $\mathrm{Di}$ is the distance and $\mathrm{Sp}$ is the wave propagation speed. In wireless data transmission, $\mathrm{Sp}=$ speed of light. This delay can be defined as a major interrupt which has to overcome in the growth of high-speed future computer technology and it is called network congestion in Integrated Circuits systems.

\section{c) Processing delay}

In the packet switched network, routers take some extra time to process the header information; this time is known as processing delay, which is the major component in network delay calculations.
Processing delays in advanced routers are usually very less (in microseconds or even less). After the processing the packet header information, the router directs the packet in the direction where the queuing delay or the auxiliary delay will be minimized.

\section{d) Packet transfer delay}

Packet transfer delay is caused due technology i.e. technology used in packet switching. There are four different sources of packet transfer delay:
1. Nodal processing
2. Queuing
3. Delay in transmission
4. Propagation delay

\section{e) Queuing in Packet Transfer Delay}

Networking delays are dependent on connection consistency and packet dropping with resending. Resending doubled the overall delay because it needs another round trip.

For upper speed in unfailing data transfer protocols the effect can be much more noteworthy.

$d e 2 e=d p r o+d q+d t r+d p$

The queuing delay dq is the total time that a packet stays in a queue at a node in the meantime, other packets which are already waiting to be transmitted.

The queuing delay is related to the transmission delay dt by the following approximate equation.

$d q=d t r * l q$

Here, lq is the average length of the queue. Queue length increases only when queue load factor exceeds 1 , without any bound (Kleinrock \& Tobagi, 1975) and dtr is delay in transmission.

\section{B. PDR (Packet Delivery Ratio)}

The router can drop a few packets if their data is destroyed or they arrive when their buffers are as of now full. PDR is the ratio of the delivered packets upon packets sent, which is directly associated with the efficiency and throughput of the network.

Packet delivery can be defined as the ratio of data packets received at the destination end in comparison to those of packet sent from the source.

$P D R=S 1 / S 2$

Where, $\mathrm{S} 1$ is the sum of all the packets received at destination and S2 is the sum of all the packets generated at the source.

\section{METHODOLOGY USED}

To achieve the goal related to the problem defined above, the network will be designed using concept defined below in the PDR and then the End-to-End Delay concept is applied to end nodes only. Here the protocol worked is AODV.

\section{A. PDR Concept}

In standard AODV the distance vector routing algorithm, i.e. Bellman-Ford algorithm is used. But in our "Improved AODV" the new concepts which are used as follows:

a) Dijkstra's algorithm

Dijkstra produces a set of Shortest Path Tree Set (SPTS) that maintains all paths of vertices included in STPS, which means 
the minimum distance from the source to destination can be calculated and the initial set is empty[14].

\section{b) Distance calculator between two nodes}

A well known Random Geometric Graph (RGG) model is used to construct the MANET and nodes with minimum distance have higher chance to get connected. Assume two arbitrary nodes $\mathrm{M}\left(\mathrm{x}_{\mathrm{m}}, \mathrm{y}_{\mathrm{m}}\right)$ and $\mathrm{N}\left(\mathrm{x}_{\mathrm{n}}, \mathrm{y}_{\mathrm{n}}\right)$ the Euclidean distance between them can be calculated as:

$$
\sqrt{ }\left(x_{n}{ }^{2}-x_{m}{ }^{2}\right)-\left(y_{n}{ }^{2}-y_{m}{ }^{2}\right)
$$

If the distance is equal to or less than the transmission range (TR) of MANET then they are said to be neighbors.

The following steps have to be followed for designing:

\section{Algorithm 1 (Improved PDR)}

1. Firstly graphical design will be done which shows the connection of the network

2. Now calculate the distance between nodes using Euclidean method. Assume two nodes are $\mathrm{M}\left(\mathrm{x}_{\mathrm{m}}, \mathrm{y}_{\mathrm{m}}\right)$ and $\mathrm{N}\left(\mathrm{x}_{\mathrm{n}}, \mathrm{y}_{\mathrm{n}}\right)$ then Euclidean distance between the two nodes will be :

$$
\sqrt{ }\left(x_{n}{ }^{2}-x_{m}{ }^{2}\right)-\left(y_{n}{ }^{2}-y_{m}{ }^{2}\right)
$$

3. Now to connect the nodes apply Dijkstra algorithm with respect to distances and prepare routing table entries to find the shortest route.

4. Now network is ready for transmission.

To generate the network at first assume that all nodes as individual as shown in Fig 2. After it the distance will be calculated between every node using Euclidean distance formula, i.e. $\sqrt{ }\left(\mathrm{x}_{\mathrm{n}}{ }^{2}-\mathrm{x}_{\mathrm{m}}{ }^{2}\right)-\left(\mathrm{y}_{\mathrm{n}}{ }^{2}-\mathrm{y}_{\mathrm{m}}{ }^{2}\right)$, refer to Fig 3 .

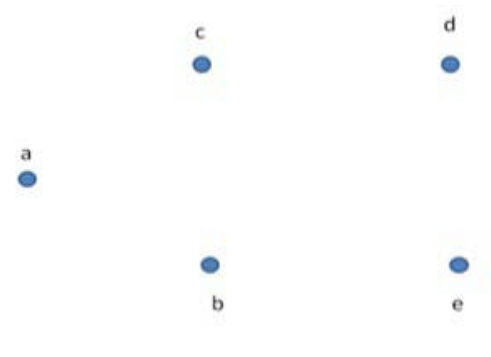

Fig 2: Individual Nodes to Form a Network

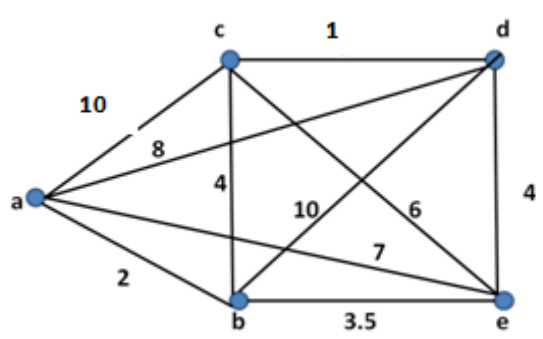

Fig 3: Nodes with Respective Distances

This process is used at the time of route discovery. With this gathered distances arrange the network in the manner to find the shortest path. The IP addressing can be done in the network in any order.

Now take an example as node "a" wants to send message to node "d", now node "a" will run the Dijkstra algorithm on the network to find the shortest path to reach the node "d".
First, node "a" will be chosen to find possible routes to any other node than choosing the shortest path to traverse it further in the direction of "d", as shown in Fig 4 and Fig 5.

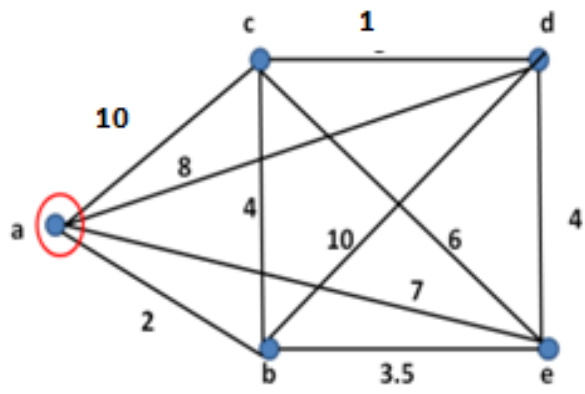

Fig 4: Network with Source as “a”

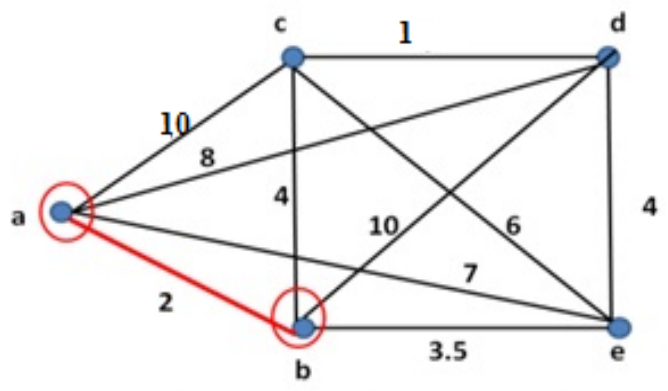

Fig 5: Traversing From a To b

Now choose the node "b" because of its minimal distance from "a”, refer to Fig 5. After it repeat the process till destination node hasn't come. Now we get the final route as a-b-c-d and distance as 7 which is minimal, refer Fig 6.

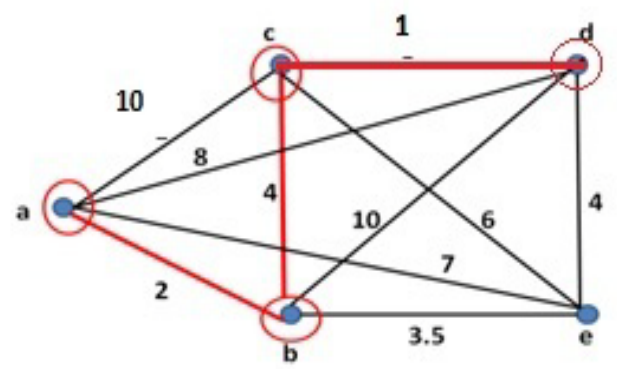

Fig 6: Final Minimal Route From a to d

\section{B. End-to-End Delay Concept}

The FCFS algorithm is simple by its process. The job served first which has come first, but it is not interruptive by its nature too, so the process which comes last will suffer from the problem of waiting if the process which is processing is taking too much time in processing. This deficiency was removed by the Shortest Job First (SJF) algorithm. SJF resolve the problem of FCFS i.e. it takes average waiting time for a set of jobs but it is also non preemptive so in case of emergency connection it fails, this problem was removed by Priority Algorithm. The priority algorithm is one which removes the deficiency of FCFS and SJF both

To remove the deficiencies of the algorithms exist for the queuing in the MANET (i.e. FCFS, SJF, and Priority Scheduling, etc.) and minimize the delay we tried to propose an algorithm.

There are different criteria which can consider while choosing the "best" scheduling algorithm for a particular purpose and 
environment for the processing of data or send the data over the network, including:

- Waiting time

- Response time

- Throughput

- Turnaround time

- CPU utilization

\section{Algorithm 2 (Improved End-to-End Delay) / Hybrid Algorithm}

First sense the channel using CSMA/CA. Through which the traffic over channel can be sensed.

Now basic four conditions will arise:

a. If the channel is free means, we can send data without any Hassel over the channel.

b. If the channel is quite busy means, we can send the but the consistency of data received will be less.

c. But if the channel is too busy means the consistency is very less.

d. Last will be sensing of the channel is failed.

2. Now apply the hybrid algorithm which is the combination of FCFS, SJF and Priority algorithm of scheduling, refer to Fig 7 [14].

\{

$$
\text { If (Channel }==\text { free) }
$$

//Apply First Come First Serve Algorithm for scheduling the processes into the channel

\}

else if(Channel $==$ busy)

\{

//Apply the SJF algorithm of scheduling for the processes.

Get the burst and arrival time of all processes and push into a buffer in increasing order with respect to burst time. Now pop the process which has lesser burst time and process it (i.e. sequentially).

\}

else if (Channel $==$ very busy)

\{

//Apply the Priority scheduling with preemption to schedule the processes.

Get the burst and arrival time and set priority of all processes and push into a buffer in increasing order with respect to priority and arrival time.

Now pop the process which has the highest priority and processes it (i.e. sequentially).

\}

else

\{

Again sense the channel

\section{\}}

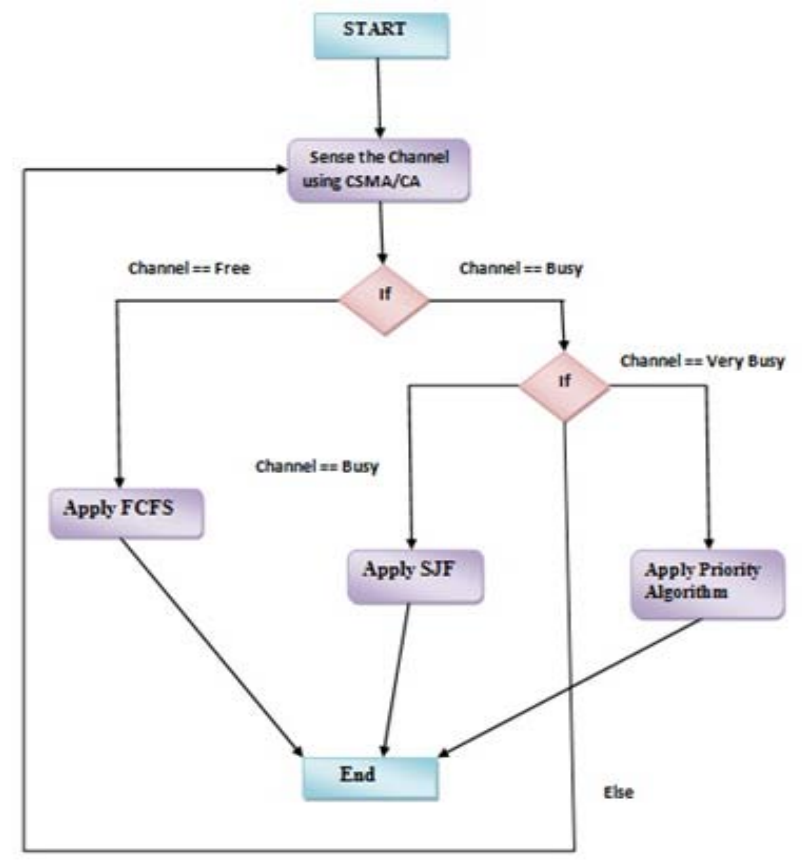

Fig 7: Flow Chart of End-to-End Delay concept

\section{SimUlatioN}

Simulation is the way to represent the operations of realworld artificially over time. The act of simulation firstly necessitates a model to be created and this model symbolizes the major and important behavior or characteristics of chose process or framework. The model integrates all sub-systems which in turn represent the system itself, whereas the simulation shows the operation of the system based on time artificially.

The main concern about the simulation includes verification of the valid source of information about the right selection of key characteristic and performance parameters, the use of simplifying, approximations and suppositions within the simulation, and validity of the simulation output.

To simulate Mobile Ad hoc Network, some parameters have to be fixed. Fix parameters are used to set an environment for simulation with some fix conditions. The parameters can be fixed like:

- Time for Simulation

- Area of Simulation

- Traffic Type

- Data Payload

- Type of Channel

- MAC Type

- Mobility

- Model of Antenna

- IFQ Length

Various network discrete event based simulators are available such as NS-2, NS-3, REAL, OMNeT++, NetSim, JSim, OPNET, and QualNet.

The comparison between different simulators has been done and after that NS2 is found as the best choice to simulate our new AODV in Mobile Ad hoc Network (Wireless environment). The reasons behind the selection are some special characteristics of NS2 and those are:

1. Topology definition: to make the simulation defines all basic parameters and have facilities to correlate them, NS-2 is one such tool which can perform all these. 
2. Model development is easier with the use of helpers.

3. Node and its link configuration with default values like the size and number of packets sent by an application or minimum transmission unit (MTU) of a point-to-point link are made using the attribute system.

4. Execution with logging of events and data.

5. Performance analysis can be statistically done with the help of tools such as R to draw conclusions.

6. Graphical Visualization of data can be created using tools like Gnuplot, matplotlib or XGRAPH of NS-2 of simulated results.

7. NS-2 supports $\mathrm{C}++$ language which is easy to understand and modify the coding.

8. It is a freely available simulator[15].

\section{A. Fixed Parameters}

To simulate the IAODV in Mobile Ad hoc Network some parameters have to be fixed. Fix parameters are used to set an environment for simulation with some constraints in conditions. Here the fixed values of these parameters to simulate our new algorithm are, as depicted in Table 1.

Table - 1 Fixed Parameters

\begin{tabular}{|c|c|}
\hline Parameter & Value \\
\hline Simulator used & NS-2.34 \\
\hline Protocols Studied & AODV and Improved AODV \\
\hline Time of Simulation & 300 seconds \\
\hline Area of Simulation & CBR \\
\hline Type of Traffic & 512 bytes/packet \\
\hline Data payload & Wireless Channel \\
\hline Type of Channel & 802.11 \\
\hline MAC type & Random \\
\hline Mobility & Omni \\
\hline Model of Antenna & 50 \\
\hline IFQ Length &
\end{tabular}

\section{ANALYSIS OF RESULT}

To improve the performance of the existing AODV:

- End-to-End Delay

- Packet Delivery Ratio

On the basis of methodology which is explained earlier, the existing standard AODV is modified [16]. A discrete eventbased simulator NS2 is used to simulate the network and analysis has been completed by comparing of "Standard AODV" and "Improved AODV". Two scenarios of 20 and 40 nodes were designed for analysis. The simulation has been done as per the fixed parameters defined in table 1 . The results of the simulation can be seen in Fig 8.

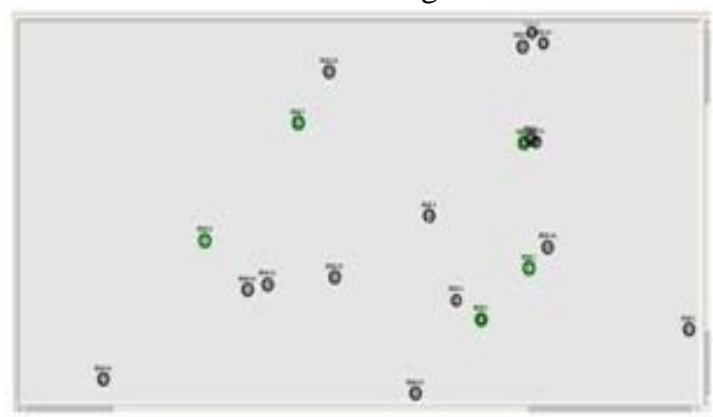

\section{A. Improvement in End-to-End Delay with 20 nodes}

The overall average improvement in End-to-End Delay in "IAODV" is approximate $7.35 \%$ and which shows End-to-End Delay has been reduced and protocol is efficient. Outcomes are shown in Graph 1, Graph 2 and Table 2.

TABLE - 2 Improvement In End-To-End Delay With 20 Nodes

\begin{tabular}{|l|l|l|l|l|}
\hline Mobility & AODV & $\begin{array}{l}\text { Improved } \\
\text { AODV }\end{array}$ & Difference & \% Improvement \\
\hline $\mathrm{m} 1$ & 219 & 233 & -14 & -6.39 \\
\hline $\mathrm{m} 2$ & 200 & 165 & 35 & 17.5 \\
\hline $\mathrm{m} 3$ & 184 & 162 & 22 & 12.43 \\
\hline $\mathrm{m} 4$ & 170 & 160 & 10 & 5.88 \\
\hline \multicolumn{5}{|c|}{ Average 7.35} \\
\hline
\end{tabular}

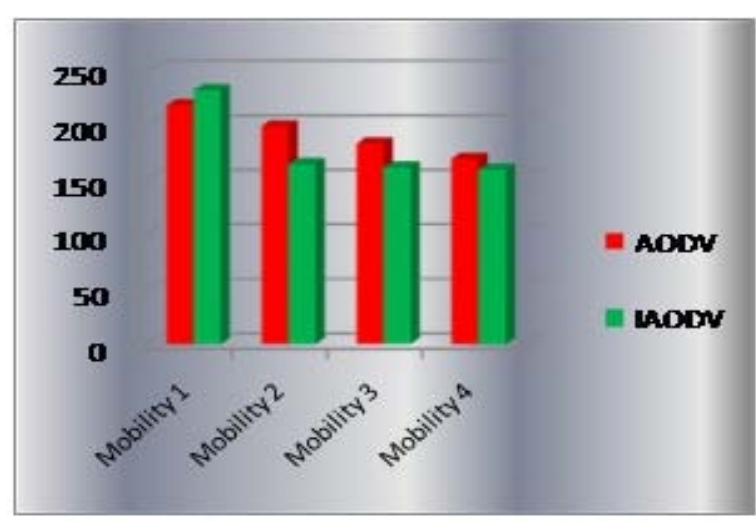

Graph 1: Improved End-to-End Delay with 20 Nodes

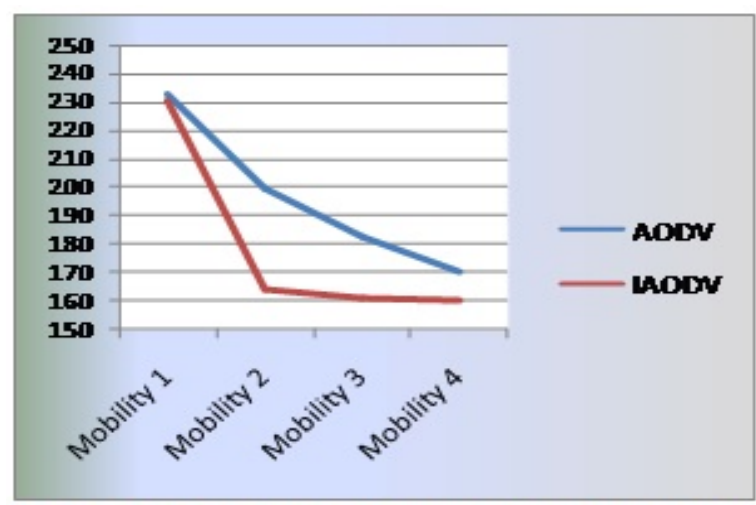

Graph 2: Improved End-to-End Delay with 20 nodes

\section{B. Improvement in End-to-End Delay with 40 nodes}

The improvement which is calculated from the data varies from $6 \%$ to $12 \%$. The average improvement in End-to-End Delay in "IAODV" is approximate $7.76 \%$ and which shows End-to-End Delay has been reduced and protocol is efficient. Results are delineated in Graph 3, Graph 4 and Table 3. 
Table - 3 Improvement in end-to-end delay with 40 nodes

\begin{tabular}{|c|c|c|c|c|}
\hline Mobility & AODV & $\begin{array}{c}\text { Improved } \\
\text { AODV }\end{array}$ & Difference & $\begin{array}{c}\text { \% } \\
\text { Improve } \\
\text { ment }\end{array}$ \\
\hline $\mathrm{m} 1$ & 218 & 231 & -13 & -5.96 \\
\hline $\mathrm{m} 2$ & 200 & 164 & 36 & 18 \\
\hline $\mathrm{m} 3$ & 183 & 161 & 22 & 12.03 \\
\hline $\mathrm{m} 4$ & 172 & 160 & 12 & 6.97 \\
\hline \multicolumn{6}{|c|}{ Average 7.76} \\
\hline
\end{tabular}

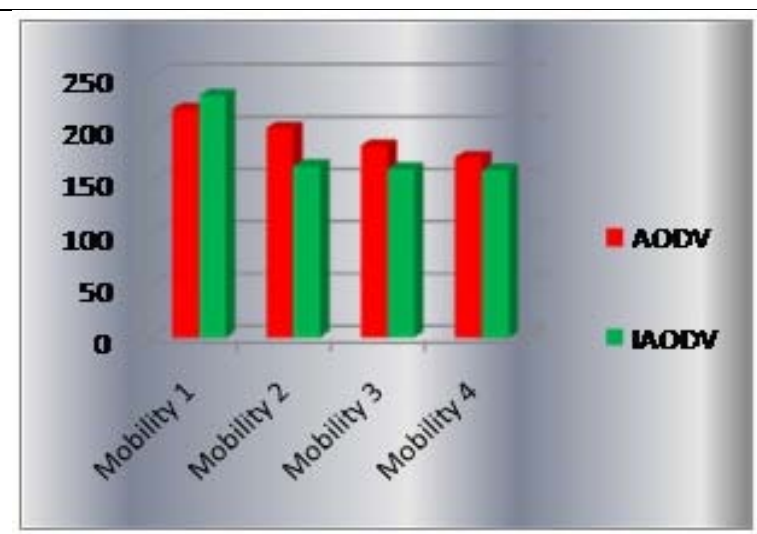

Graph 3: Improved End-to-End Delay With 40 nodes

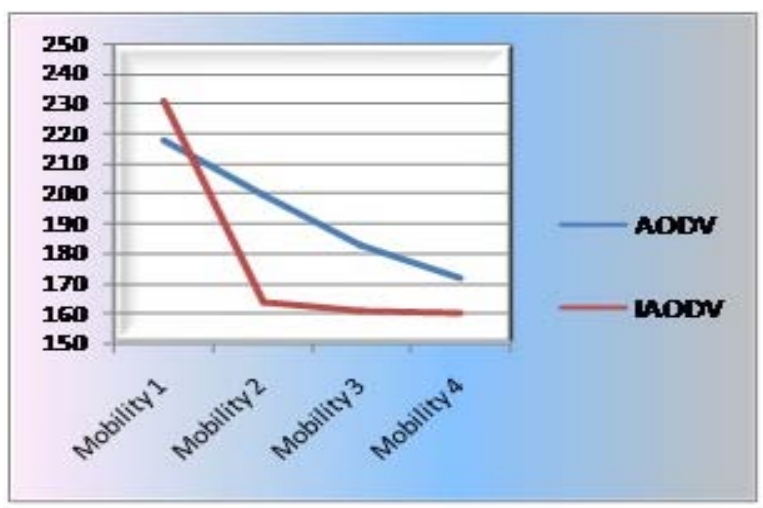

Graph 4: Improved End-to-End Delay with 40 nodes

\section{Improvement in PDR with 20 nodes}

The improvement which is calculated from the data varies from $1.6 \%$ to $5.19 \%$. The average improvement in Packet Delivery Ratio in "IAODV" is approximate 2.8\% and which shows Packet Delivery Ratio has been improved and protocol is efficient. Outcomes are portrayed in Graph 5, Graph 6 and Table 4.

Table - 4 Improvement In PDR With 20 Nodes

\begin{tabular}{|l|l|l|l|l|}
\hline Mobility & AODV & $\begin{array}{l}\text { Improved } \\
\text { AODV }\end{array}$ & Difference & \% Improvement \\
\hline $\mathrm{m} 1$ & 75 & 77 & 2 & 2.66 \\
\hline $\mathrm{m} 2$ & 77 & 81 & 4 & 5.19 \\
\hline $\mathrm{m} 3$ & 79.5 & 80.9 & 1.4 & 1.76 \\
\hline $\mathrm{m} 4$ & 81 & 82.3 & 1.3 & 1.60 \\
\hline \multicolumn{5}{|c|}{ Average 2.80} \\
\hline
\end{tabular}

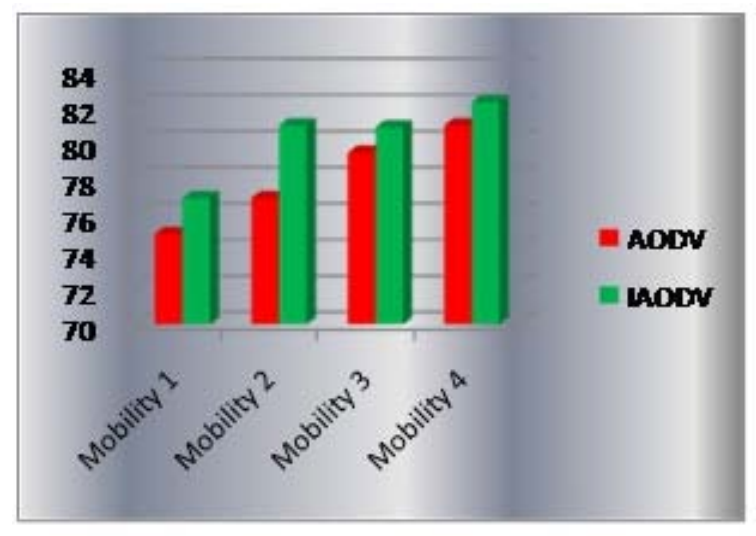

Graph 5: Improved PDR With 20 Nodes

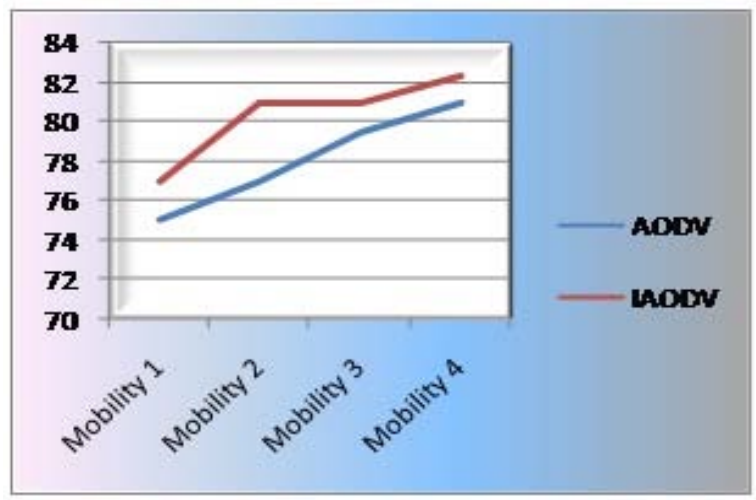

Graph 6: Improved PDR With 20 Nodes

\section{Improvement in PDR with $\mathbf{4 0}$ nodes}

The improvement which is calculated from the data varies from $1.5 \%$ to $4.53 \%$. The average improvement in Packet Delivery Ratio in "IAODV" is approximate $2.445 \%$ and which shows Packet Delivery Ratio has been improved and protocol is efficient. Fallouts are delineated in Graph 7, Graph 8 and Table 5.

Table - 5 Improvement In PDR With 40 Nodes

\begin{tabular}{|c|c|c|c|c|}
\hline Mobility & AODV & $\begin{array}{c}\text { Improved } \\
\text { AODV }\end{array}$ & Difference & \% Improvement \\
\hline $\mathrm{m} 1$ & 74.9 & 76.5 & 1.6 & 2.13 \\
\hline $\mathrm{m} 2$ & 77.1 & 80.6 & 3.5 & 4.53 \\
\hline $\mathrm{m} 3$ & 79.5 & 80.7 & 1.2 & 1.50 \\
\hline $\mathrm{m} 4$ & 80 & 81.3 & 1.3 & 1.62 \\
\hline \multicolumn{5}{|c}{ Average 2.445} \\
\end{tabular}

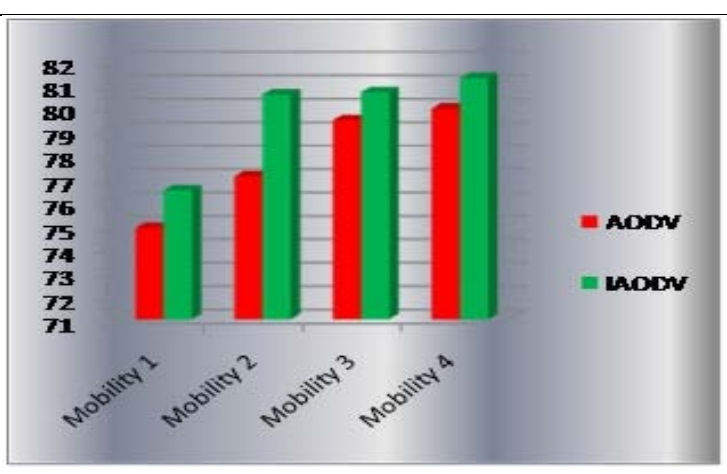

Graph 7: Improved PDR With 40 Nodes 


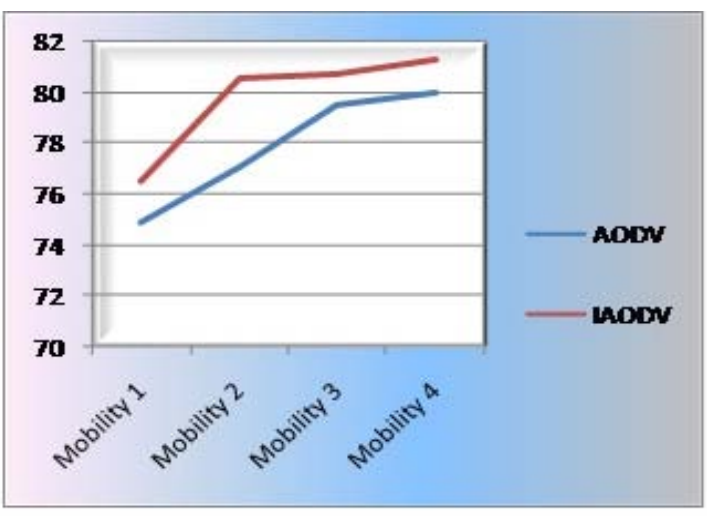

Graph 8: Improved PDR With 40 Nodes

\section{CONCLUSION}

Progress in mobile computing and wireless network technologies are gaining the attention of researchers and also stimulating possibilities of the future shape of wireless mobile ad hoc network technologies. This Wireless Network is made up of mobile devices which can freely move in any direction. The problem in MANET is to deal with the transmission of the packet across the network from source to destination. To overcome on aforementioned problems, the new protocol is developed named Improved AODV (IAODV). The overall efficiency of the network is improved in terms of "Improved AODV". The outcome of "Improved AODV" enhances the overall efficiency in terms of reduced "End-to-End Delay" of the packet and improved "Packet Delivery Ratio" and overall throughput is also increased because the delivery ratio of the packets is increased.

\section{FUTURE SCOPE}

In mobile computing and wireless network technologies, advancements are producing the exciting results for the future. MANET faces many challenges like a limited battery, node mobility; link lost, etc. which affects the network performance. In future there is a lot of scope to work on these challenges to improve the overall performance and efficiency of the network. This newly developed IAODV has to be tested with more numbers of nodes and higher data transfer for a highspeed network.

\section{REFERENCES}

[1] Meshram, S. J., Khobragade, R. N., \& Thakare, V. M. (2015). Bandwidth Prediction in Multichannel MANET for Improving Quality of Service. International Journal of Advanced Research in Computer Science and Software Engineering,5(4), 47-50.

[2] Simaremare, H., Abouaissa, A., Sari, R. F., \& Lorenz, P. (2014). Performance analysis of optimized trust AODV using ant algorithm. 2014 IEEE International Conference on Communications (ICC). doi:10.1109/icc.2014.6883591.
[3] Ankur (1970, January 01). MANETs. Retrieved May 10, 2017, http://manets.blogspot.in/2007/01/definition.html.

[4] Malini, S., Kannan, E., \& Valarmathi, A. (2012). Performance optimization of single-path and multi-path AODV using response surface method (RSM). In (pp. 1-4). USA: IEEE. doi:10.1109/SECon.2012.6196895.

[5] Singh, H., Kaur, H., Sharma, A., \& Malhotra, R. (2015). Performance Investigation of Reactive AODV and Hybrid GRP Routing Protocols under Influence of IEEE 802.11n MANET. 2015 Fifth International Conference on Advanced Computing \& Communication Technologies. doi:10.1109/acct.2015.50.

[6] Ahmad, I., Ashraf, U., Anum, S., \& Tahir, H. (2014). Enhanced AODV Route Discovery and Route Establishment for QoS Provision for Real-Time Transmission in MANET. International Journal of Computer Networks \& Communications,6(2), 79-87. doi:10.5121/ijcnc.2014.6207.

[7] Alsmira, M. A., Jararweh, Y., Obaidat, I., \& Gupta, B. B. (2017). Internet of surveillance: a cloud-supported largescale wireless surveillance system. Journal of Supercomputing. doi:10.1007/s11227-016-1857-x.

[8] Stergiou, C., Psannis, K. E., Kim, B., \& Gupta, B. (2016). Secure integration of IoT and Cloud Computing. Future Generation Computer Systems. doi:10.1016/j.future.2016.11.031.

[9] Tewari, A., \& Gupta, B. B. (2016). Cryptanalysis of a novel ultra-lightweight mutual authentication protocol for IoT devices using RFID tags. The Journal of Supercomputing,73(3), 1085-1102. doi:10.1007/s11227-0161849-x.

[10] G. (1970, January 01). Mobile ad hoc network. Retrieved April 05, 2017, from http://mobileeAdhocnetwork.blogspot.in/2012/02/types-ofmanet.html

[11] Singh, T. Y., \& Reddy, K. S. (2015). Enhancing The QoS Using Aodv And Dsdv Protocols In Manets Based On Energy Consumption. Global Journal For Research Analysis,4(5), 1-2.

[12] Corson, S., \& Macker, J. (1999). Mobile Ad hoc Networking (MANET): Routing Protocol Performance Issues and Evaluation Considerations. ACM

[13] Kleinrock, L., \& Tobagi, F. (1975). Packet Switching in Radio Channels: Part I--Carrier Sense Multiple-Access Modes and Their Throughput-Delay Characteristics. IEEE Transactions on Communications,23(12), 1400-1416. doi:10.1109/tcom.1975.1092768.

[14] Shrivastava M., Sahu M., Matele S., \& Rizvi M. A. (2013). Improving Efficiency of MANET by Reducing Queuing Delay Using Hybrid Algorithm. International Journal of Advanced Research in Computer Science,4(8), 338-344.

[15] Negus, K., Stephens, A., \& Lansford, J. (2000). HomeRF: wireless networking for the connected home. IEEE Personal Communications,7(1), 20-27. doi:10.1109/98.824568.

[16] Adam S. M., \& Hassan R. (2013). Delay aware Reactive Routing Protocols for QoS in MANETs: a Review. Journal of Applied Research and Technology,11(6), 844-850. doi:10.1016/s1665-6423(13)71590-6. 